\title{
Effects of Anode Nozzle Geometry on Ambient Air Entrainment Into Thermal Plasma Jets Generated by Nontransferred Plasma Torch
}

\author{
Sooseok Choi, Tae Hyung Hwang, Jun Ho Seo, Dong Uk Kim, and Sang Hee Hong, Member, IEEE
}

\begin{abstract}
The geometrical effects of an anode nozzle in a nontransferred plasma torch on air entrainment are examined by measurements of plasma composition using a quadruple mass spectrometry. In addition, the radial and axial distributions of plasma enthalpy, temperature, and velocity are measured by using an enthalpy probe method. Two types of anode nozzle geometry, i.e., cylindrical and stepped nozzles, are employed for the torch in this experiment. As a result of gas composition measurements, the new stepped nozzle turns out to produce a thermal plasma jet having lower air content in it compared with the conventional cylindrical nozzle. The plasma jet produced by the stepped nozzle exhibits higher enthalpy and temperature, especially around the core of the plasma flame, due to less intrusion of ambient air. Furthermore, the axial velocity distribution with a slowly changing variation is observed in the stepped nozzle case because of the plasma flow less disturbed by air entrainment. From these experimental results of thermal plasma characteristics and nozzle geometry effects on air entrainment, a high quality of coating products are expected in plasma spraying by using the stepped nozzle due to higher plasma enthalpy and temperature and lower velocity drop along the plasma jet.
\end{abstract}

Index Terms-Air entrainment, enthalpy probe, nozzle geometry effects, plasma spraying, plasma torch, quadruple mass spectrometry.

\section{INTRODUCTION}

I N GENERAL, higher enthalpy, temperature, and velocity of thermal plasma jets are preferable in plasma spraying for producing a high quality of coatings for their industrial uses, because coating powders are melted and accelerated in the plasma flame ejected from a nontransferred arc torch through ambient air toward the substrate. But, in the typical plasma spray process operated under an atmospheric-pressure condition, the entrainment of ambient air into the thermal plasma jet is inevitable. An air inflow to the flame alters chemical compositions of plasma species, cools the thermal plasma flame, and reduces the jet velocity [1]. Therefore, the ambient air entrainment should be controlled to a lower degree for getting better quality of coating products with high purity, density, and bond strength in the plasma spray process.

A wide variety of plasma spraying methods have been suggested to avoid an unpleasant intrusion of air into the plasma jet. The typical methods used are the low pressure

Manuscript received August 8, 2003; revised November 26, 2003.

The authors are with the Department of Nuclear Engineering, Seoul National University, Seoul 151-742, Korea (e-mail: hongsh@snu.ac.kr).

Digital Object Identifier 10.1109/TPS.2004.826365

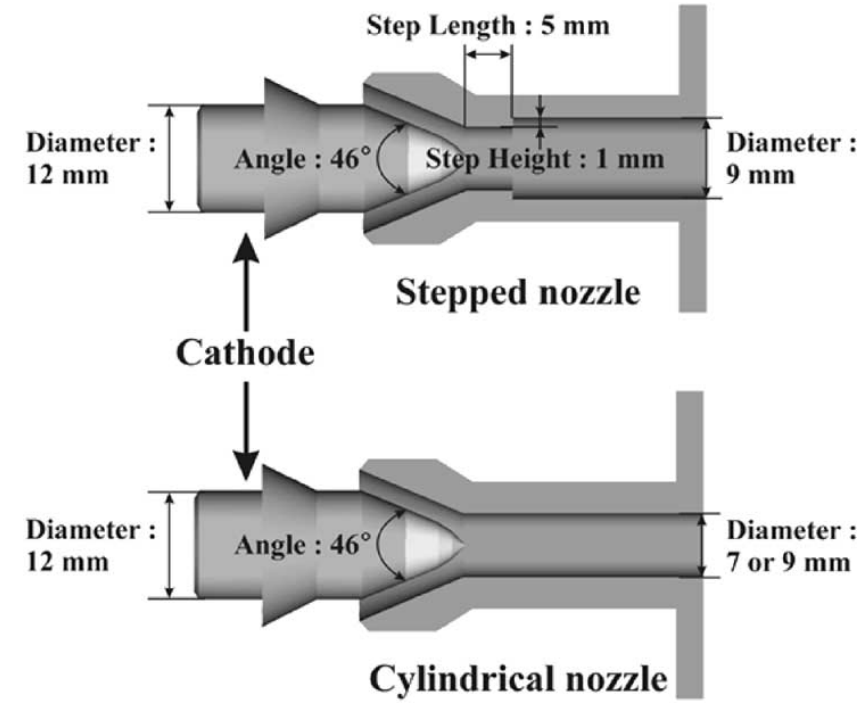

Fig. 1. Geometry of a new stepped nozzle and a conventional cylindrical nozzle.

plasma spraying (LPPS), the torch operation in an inert or nonreactive gas environment [2], and the shrouding of the plasma jet. Although these methods may control the entrainment of ambient air, they require complex equipment and high cost. Since the former two methods require a complicated system such as large chamber and pumping or gas supply facility, they demand high costs of maintenance and operation. Similarly, in the latter shrouding method, shroud gas injection devices and additional gas expenses are required [3], [4]. As an alternative method suggested in this work, modification of nozzle design, which requires nozzle cutting cost only, would be a simple and economical method. It is, therefore, essential to study the geometrical effects of nozzle shape on the ambient air entrainment for an optimal design of nozzle electrode.

Fig. 1 describes the geometry of a new stepped nozzle and a conventional cylindrical nozzle, which has been considered in this experimental work. The employment of the stepped nozzle was originally intended to stabilize the thermal plasma jets. The stepped nozzle enhances turbulent effects due to a flow separation appearing behind the nozzle step, and the radial temperature distribution and stagnation region of plasma flow are expanded toward the nozzle wall. As a result of these flow characteristics in the stepped nozzle case, an injected cold plasma gas is disturbed along the inner nozzle wall to form a dielectric layer. Therefore, the breakdown voltage between the arc column and 


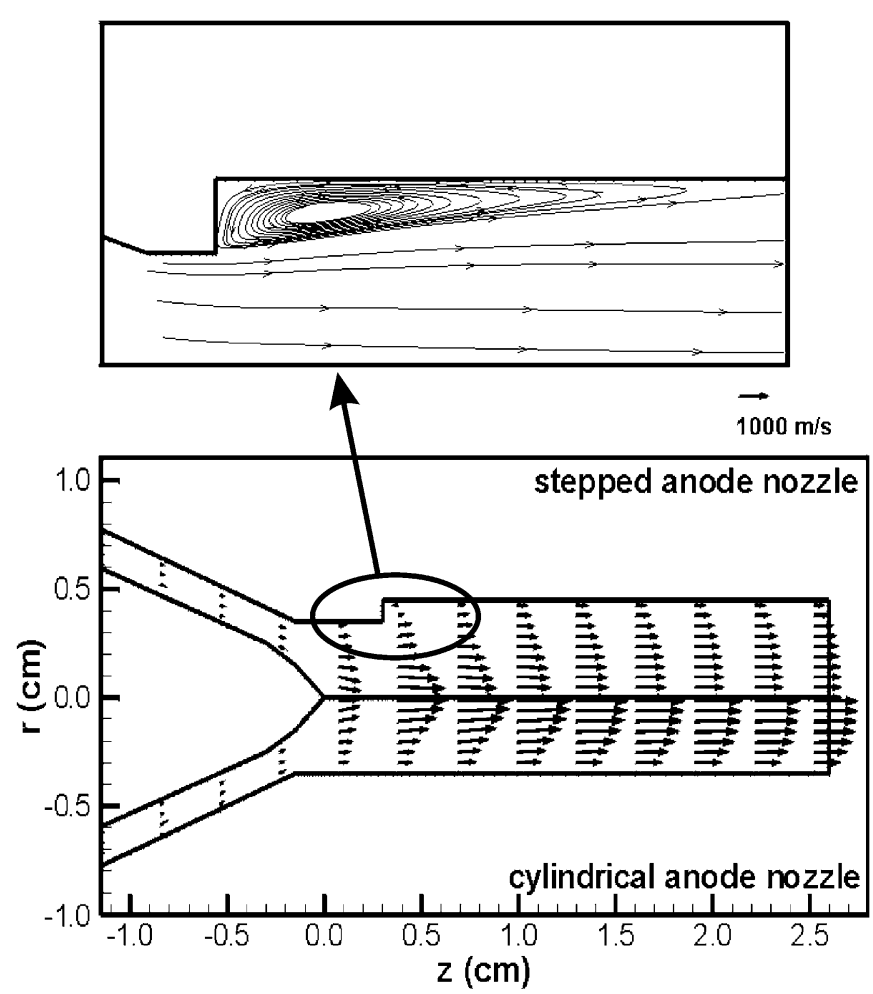

Fig. 2. Calculated stream lines and velocity vectors simulated numerically in a plasma torch with a stepped anode nozzle compared with a cylindrical anode nozzle.

the nozzle wall reduces, and the arc shunting occurs easily in a restricted region behind the nozzle step. Consequently, the amplitudes of voltage fluctuations decrease and the plasma jet is stabilized by means of this stepped nozzle. Although the stepped nozzle induces the arc attachment in the restricted region, erosion of the stepped nozzle is expected to be less severe than that of the cylindrical one. Because the arc roots restriction could be controlled for extending the arc length in the stepped nozzle, the increased arc voltage leads to the lowered arc current, in turn the reduced erosion, when the same electric power is supplied to the plasma torch. In this case, the resultant reduced arc current may result in an increase in the thermal efficiency of the torch with a stepped anode nozzle.

The entrainment of ambient air is strongly affected by a steep laminar shear of plasma jet around the nozzle exit [5], [6]. In a peripheral region of plasma jet, this large velocity difference causes a rolling up of the shear layer flow with a large-scale entrainment of ambient air [7]. Therefore, it is required that the velocity gradient at the outer edge of the plasma jet should be reduced for a small-scale air intrusion. In our previous numerical analyses shown in Fig. 2, the enhanced turbulent effects have been observed in the stepped nozzle by a flow separation behind the nozzle step, and plasma velocity fields are different between the two nozzle cases. The velocity gradient in the peripheral region of plasma jet produced in the stepped nozzle is smaller than that in the cylindrical nozzle at the nozzle exit. Therefore, it is expected that there is a small-scale entrainment of ambient air in the stepped nozzle case compared with the cylindrical one.

In this experimental work, the geometrical effects of anode nozzle on air entrainment for the nontransferred plasma torch are examined. And, for the purpose of determination of nozzle geometry suitable for producing high quality coatings, the characteristics of plasma jets produced by the stepped and cylindrical nozzles are measured. We have used a quadruple mass spectrometry (QMS) to find the gas compositions of plasma jets, which affect thermal plasma characteristics. An enthalpy probe method has been used to measure enthalpy, temperature, and velocity of plasma jets produced by each of the two nozzles.

\section{EXPERIMENTAL SETUP}

A schematic diagram of an enthalpy probe diagnostics system is presented in Fig. 3. The enthalpy probe diagnostics system consists of several different pieces of equipment for measurements of gas composition, enthalpy, temperature, and velocity of plasma jets.

A residual gas analyzer (Stanford Research Systems RGA200) is used for the QMS to measure gas composition of plasma jets [8]. Three $K$-type thermocouples measure the temperatures of inlet and outlet coolant and sampling gas, respectively. Flow rates are measured by a flow meter for coolant and by a mass flow controller (MFC) for sampling gas, respectively. Using the measured values from this diagnostics system, we can find out the enthalpy of plasma jet from the following:

$$
h_{i}=h_{e}+\frac{\dot{m}_{c}}{\dot{m}_{g}} C_{p}\left[\left(\Delta T_{c}\right)_{\text {gas flow }}-\left(\Delta T_{c}\right)_{\text {no gas flow }}\right]
$$

where $h_{i}$ represents the unknown gas enthalpy at the probe entrance, and $h_{e}$ is the gas enthalpy at the probe exit. But, $h_{e}$ is normally negligible since it is known to be less than $0.1 \%$ of $h_{i}$ [9]. $\dot{m}_{c}$ and $\dot{m}_{g}$ are the mass flow rates of cooling water and sampling gas, respectively, and $C_{p}$ is the specific heat of water. $\left(\Delta T_{c}\right)_{\text {gas flow }}$ and $\left(\Delta T_{c}\right)_{\text {no gas flow }}$ are the temperature rises of cooling water for the sampling and tare measurements, respectively. Taking advantage of the plasma enthalpy obtained in this way, we can determine the temperature of plasma jet by using a tabulated data based on the local thermodynamic equilibrium (LTE) [10]. During the tare measurement, the pressure difference between plasma and atmosphere can be measured by a differential pressure gauge. Since the probe may be used as a Pitot tube, the plasma velocity can be calculated from the Bernoulli equation

$$
U=\sqrt{\frac{2\left(p_{0}-p_{a}\right)}{\rho(T)}}
$$

where $p_{0}$ and $p_{a}$ are the pressures of plasma jet and atmosphere, respectively, and $\rho(T)$ is the mass density of plasma jet flow at the local plasma temperature.

The residual gas analyzer can be calibrated by using dry air, because it has fixed composition fractions of gases such as nitrogen, oxygen, and argon. After the calibration, a known nitrogen-argon mixture is supplied to the enthalpy probe through an electronic mass flow controller. In this experimental work, an uncertainty of $2.6 \%$ was obtained in the gas composition measurements on the thermal plasma jet, which is good agreement with the uncertainty in its natural nitrogen-argon mixture 


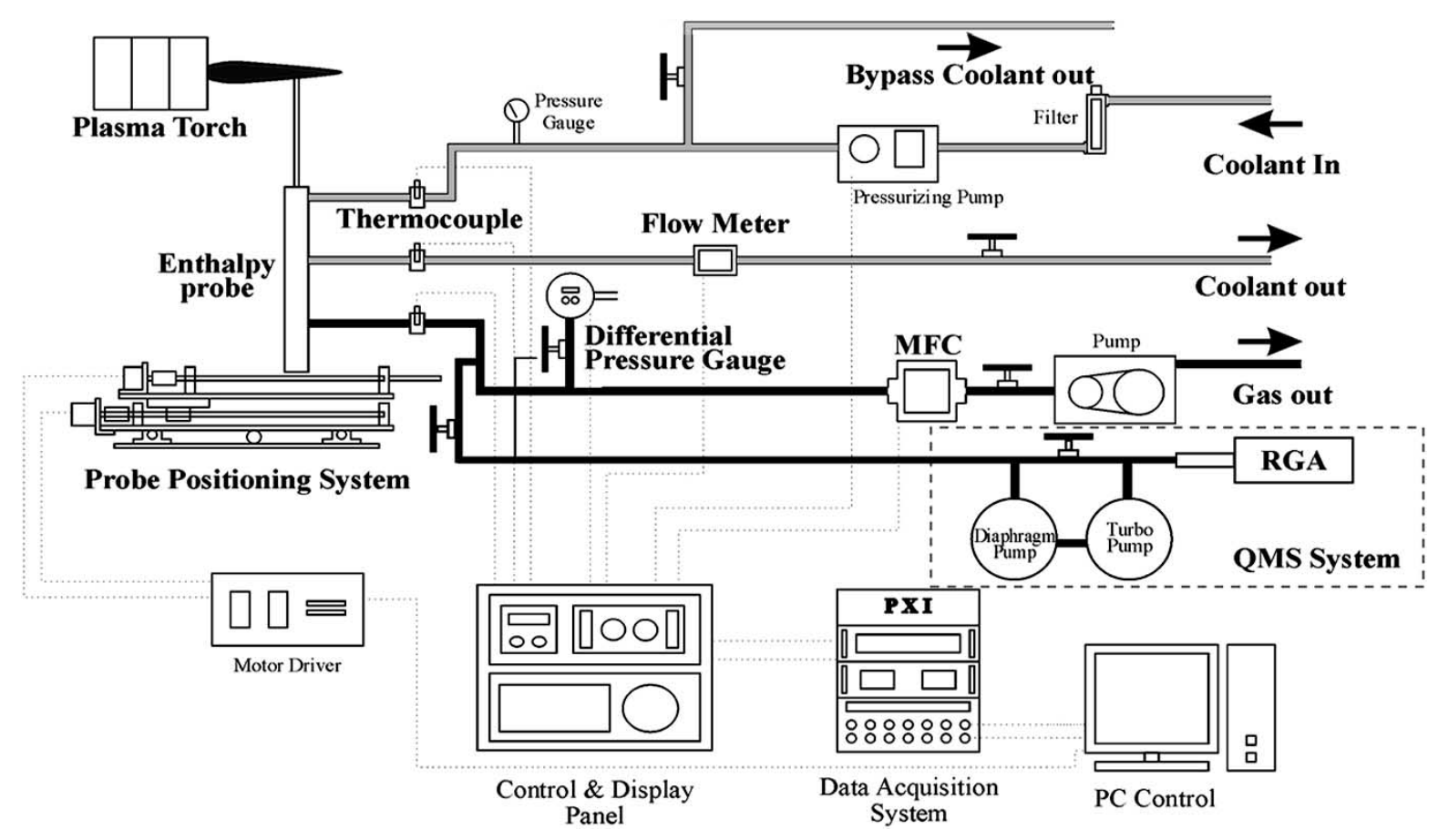

Fig. 3. Schematic diagram of an enthalpy probe diagnostics system with a QMS system used for this experiment.

of $2.4 \%$. The uncertainty analyses on the measured enthalpy, temperature, and velocity are also carried out based on the standard error analysis technique [9]. Using this technique, the uncertainty in the measured enthalpy is estimated by the errors coming from all measurement devices as follows:

$$
\begin{aligned}
\left(\frac{\delta h}{h}\right)^{2} & =\left(\frac{\delta \dot{m}_{c}}{\dot{m}_{c}}\right)^{2}+\left(\frac{\delta \dot{m}_{g}}{\dot{m}_{g}}\right)^{2} \\
& +\left(\frac{\delta T}{\left(\Delta T_{c}\right)_{\text {gas flow }}-\left(\Delta T_{c}\right)_{\text {no gas flow }}}\right)^{2} .
\end{aligned}
$$

In this experiment, the error associated with the measurement of the cooling water mass flow rate was $7.1 \%$. The mass flow controller was able to provide the gas mass flow rate with an accuracy of $5.2 \%$. The absolute error $\delta T$ of temperature difference between the gas sampling and tare measurements was $0.08{ }^{\circ} \mathrm{C}$, because the temperatures of cooling water flow were measured within $\pm 0.02{ }^{\circ} \mathrm{C}$. The average difference of temperature rise between the sampling and tare measurements in all measured points was $0.93{ }^{\circ} \mathrm{C}$. Thus, the error associated with the coolant temperature measurement was $8.6 \%$. The uncertainty in the measured plasma enthalpy is finally estimated to be $12.3 \%$, and this uncertainty is also applicable to the temperature measurement with the assumption of LTE since it is calculated by the tabulated data. The error associated with the differential pressure gauge was $10.8 \%$, and in the same manner as in the uncertainty calculation of measured enthalpy, the uncertainty in the plasma velocity measurement was estimated as $8.6 \%$. These uncertainty values have been calculated for the average plasma enthalpy and velocity values in the zone where the measurements were made. Therefore, relatively low uncertainties would be associated with the enthalpy probe measurements in the high-temperature region of plasma jet. On the contrary, relatively high uncertainties would be measured in the low temperature region of plasma jet.

In the present measurements, an input power supplied to the nontransferred torch was $15 \mathrm{~kW}$ and a fixed argon flow rate of $40 \mathrm{slpm}$ was used as a plasma gas. We have employed three kinds of anode nozzle described in Fig. 1: a stepped nozzle with 7 to $9 \mathrm{~mm}$ diameters and two cylindrical nozzles with 7 and 9 $\mathrm{mm}$ diameters, respectively.

We have selected the measurement points where substrates are normally placed in the plasma spray process, and the melting condition of the enthalpy probe by the heat load from plasma jets has been also taken into account. The measurement points in the axial direction are located at intervals of $5 \mathrm{~mm}$ from 20 to $30 \mathrm{~mm}$ away from the nozzle exit, and those in the radial direction are at every $1 \mathrm{~mm}$ from 0 to $8 \mathrm{~mm}$ for each axial position. In order to validate the gas compositions, enthalpies, temperatures, and velocities measured for the thermal plasma flame in this experiment, the net powers determined from these measured values at each axial position are compared with the plasma jet powers deduced by the subtraction of heat removal by cooling water from the electric power supplied to the nontransferred torch.

\section{RESULTS AND DisCUSSION}

The plasma jet produced by the cylindrical nozzle is rather a laminar flow near the nozzle exit region. On the other hand, the stepped nozzle produces turbulent flows around the edge region of plasma jets caused by a flow separation existing behind the nozzle step. The jets resulting from laminar boundary layers are generally found to have faster mixing rates than the jets from turbulent boundary layers [7], [11]. Therefore, we can expect that the stepped nozzle has a positive effect to prevent the inflows of ambient air. Our experimental results for the gas compositions of the plasma jet will demonstrate this expectation of low en- 


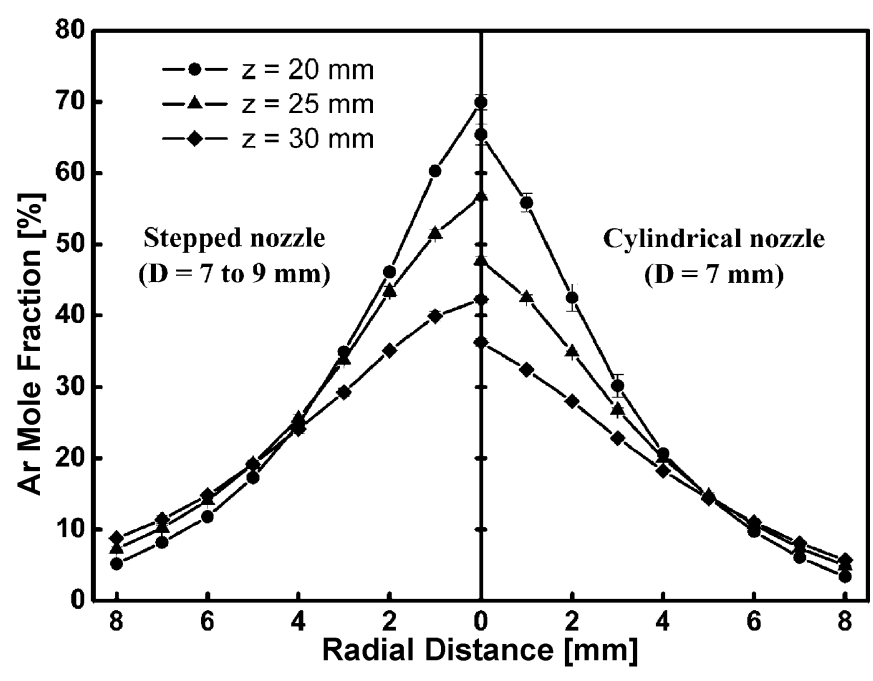

Fig. 4. Argon mole fraction profiles in the plasma jets produced by the stepped and cylindrical nozzles, respectively.

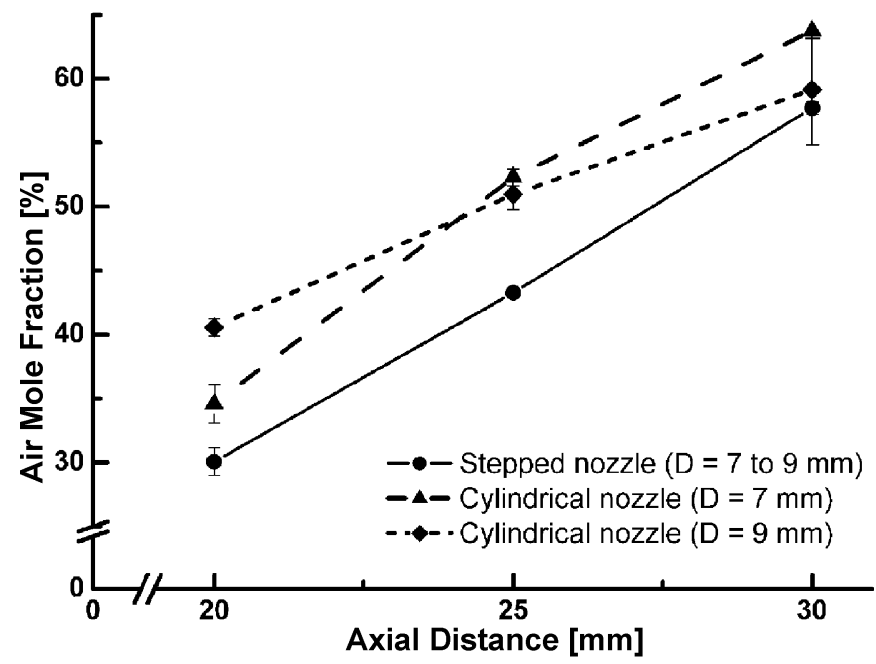

Fig. 5. Air mole fractions at the core of plasma jets in the stepped and cylindrical nozzle cases.

trainment of ambient air by using the stepped nozzle. In order to estimate what fraction of air is mixed with argon plasma, we have measured the gas compositions of plasma jet. In Fig. 4, the argon mole fractions in the plasma jet for the stepped nozzle are compared with those for the cylindrical nozzle of $7 \mathrm{~mm}$ at all measurement positions. In both the radial and axial directions, the argon composition of plasma jet is preserved higher in the stepped nozzle case than in the cylindrical case. This measured result indicates that the original characteristics of thermal plasma jet, such as high enthalpy, temperature, and velocity, could be well conserved by using the stepped nozzle, because air entrainment is less severe in the stepped nozzle case. Straightforward measurements of air mole fraction at the core $(r=0)$ of plasma flame are shown in Fig. 5. The stepped nozzle yields the lower air mole fractions invading the core of plasma jet than the cylindrical nozzle. The velocity gradients in the peripheral region of plasma jet produced by the stepped nozzle are smaller than those by the cylindrical nozzle, due to the turbulent flow effects generated in the plasma edge region by using the stepped nozzle. For this reason, entrained air contents are reduced in the

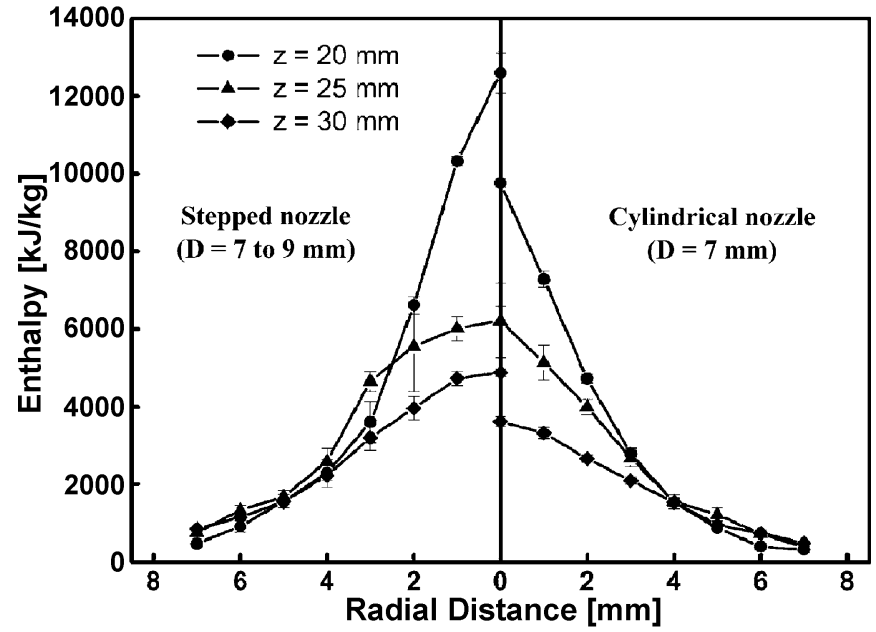

Fig. 6. Enthalpy profiles in the plasma jets produced by the stepped and cylindrical nozzles, respectively.

stepped nozzle case compared with the cylindrical case, and the stepped nozzle is then expected to generate the thermal plasma flame of higher enthalpy, temperature, and velocity.

The enthalpy profiles in the plasma jets produced by the stepped and cylindrical nozzles are presented in Fig. 6, respectively. Similar to the argon mole fractions in Fig. 4, higher enthalpies of the thermal plasma are measured in the stepped nozzle case due to the plasma flame less influenced by the entrained air. In the core region of the plasma jet at an axial position of $20 \mathrm{~mm}$, the stepped nozzle has revealed an excess of plasma enthalpy by about $2500 \mathrm{~kJ} / \mathrm{kg}$ over the cylindrical one. But, near the edge region of plasma flame, no such considerable difference in the plasma enthalpy is seen in both the stepped and cylindrical cases, because the edge plasmas are in contact with the ambient air continuously in both the nozzle geometries. On the other hand, the mixing air is transported to the core region through the entrainment process around the nozzle exit [5], [12], and there is less entrained air in the plasma jet for the stepped case than for the cylindrical case. For this reason, the plasma enthalpy in the core region is higher in the stepped nozzle case.

Fig. 7 shows the temperatures in the core region of the plasma flame for each nozzle case, of which dependency on the nozzle geometry and axial position has a tendency to follow the results of enthalpy measurements shown in Fig. 6. For all over the measurement points in the core plasma, the stepped nozzle case shows higher plasma temperatures than the cylindrical nozzle cases of 7 and $9 \mathrm{~mm}$. This result indicates that the plasma core less influenced by the entrained cold air can be formed when the stepped nozzle is used as an anode for the nontransferred plasma torch. According to the distributions of enthalpy and temperature obtained in the stepped case, a high efficiency of plasma spraying is achievable by the sprayed coating powders melted fully in the hot plasma core with high enthalpy and temperature [13].

The measured velocity profiles for the stepped and cylindrical nozzles are presented in Fig. 8, and the velocity decrease rates at the core of plasma jets between 20 and $30 \mathrm{~mm}$ from the nozzle exit are plotted in Fig. 9. The plasma jet velocity at the nozzle 


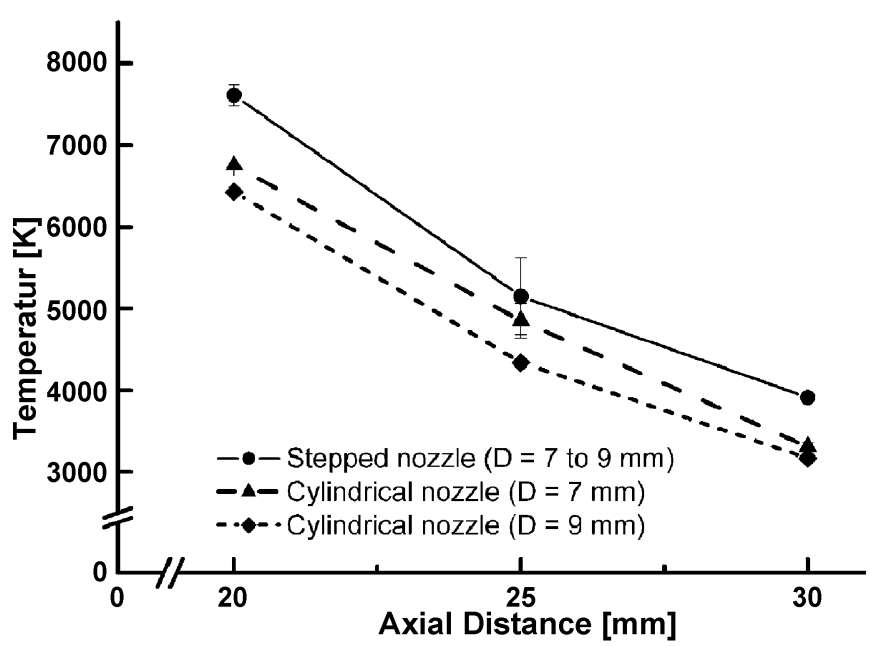

Fig. 7. Plasma temperatures at the core of plasma jets in the stepped and cylindrical nozzle cases.

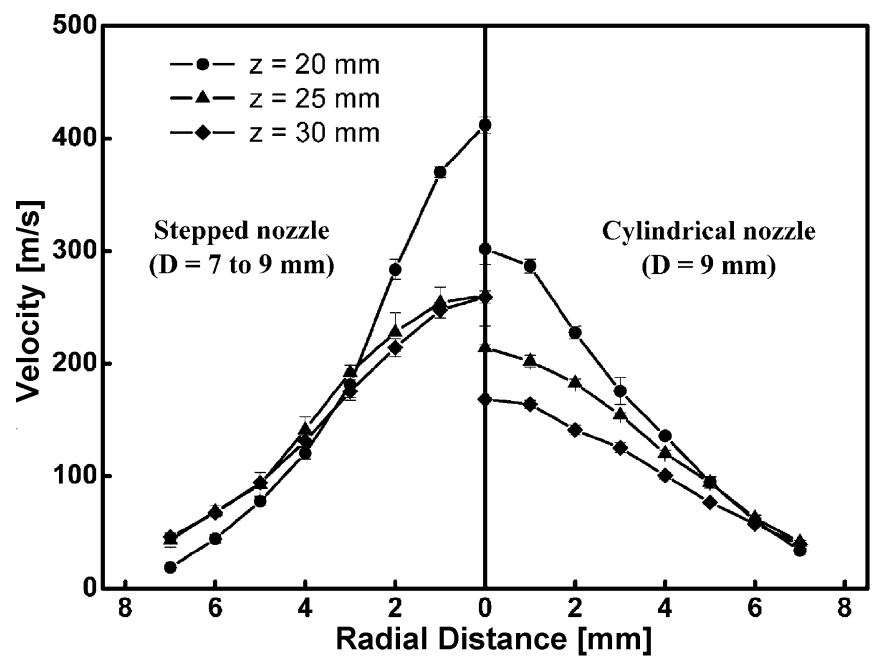

Fig. 8. Velocity profiles in the plasma jets produced by the stepped and cylindrical nozzles, respectively.

exit is basically affected by the flow rate of the plasma gas injected into the torch and by the degree of thermal expansion depending on the inner diameter of nozzle exit while the heat load of plasma jet is maintained uniform. In this experiment, the plasma velocity at the nozzle exit has been determined by the inner diameter of nozzle exit, because a fixed argon flow rate of $40 \mathrm{slpm}$ is kept as a plasma gas. Although the stepped nozzle has the same inner diameter at the nozzle exit as the cylindrical nozzle of $9 \mathrm{~mm}$, the former produces the jet velocities higher than the latter as seen in Fig. 8. The relatively higher velocities in the plasma jet for the stepped nozzle are the result of the less disturbed flow by the ambient air entrainment. As the entrainment of air increases, the centerline velocity of plasma jet decreases. Therefore, as noticed in Fig. 9, the plasma velocity for the stepped nozzle falls less slowly along the flame axis compared with that for the cylindrical one due to the relatively less disturbed flow by air entrainment. In Fig. 9, the velocity decrease rate is $37 \%$ in the stepped nozzle case, while it reaches up to $44 \%$ in the cylindrical nozzle case. It is expected from the result of velocity distributions obtained in the stepped case that the

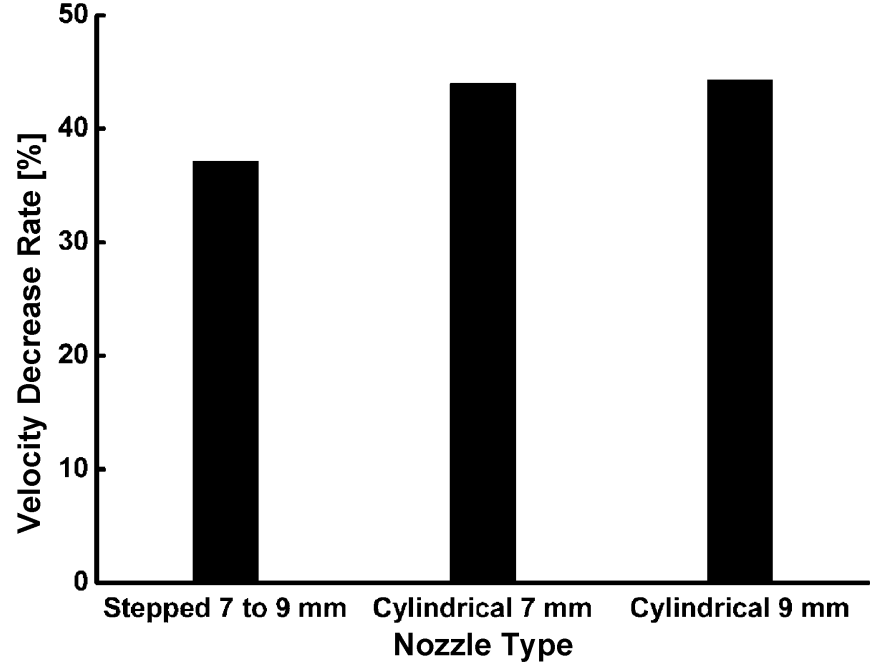

Fig. 9. Decrease rates of plasma velocity along the centerline of plasma jet between 20 and $30 \mathrm{~mm}$ away from the nozzle exit.

deposition efficiency and bond strength of the coating product will be enhanced in the plasma spraying with the stepped nozzle due to the coating powders accelerated rapidly in the fast plasma jet toward the substrate [13].

The average Reynolds numbers at both nozzle exits can be calculated on the basis of an experimental work done by Fincke et al. [14], who suggested that the shear layer is turbulent at the torch exit when the average Reynolds numbers are greater than about 400. In our experimental work, the average Reynolds numbers appear to be 613 and 596 for the stepped and cylindrical nozzles, respectively, in the case of the same nozzle exit diameter of $9 \mathrm{~mm}$. These numbers clearly indicate that the stepped nozzle enhances turbulent effects as mentioned earlier in Section I.

The disturbance of jet swirling by the nozzle step effect is expected to be profitable for the more homogeneous treatment of particles in a less divergent particle spray jet. In an effort to improve uniform particle heating, Chen et al. modified the commercial dc plasma spray torch to introduce an additional anti-vortex gas flow [13]. According to their results, the antivortex gas flow tends to keep the particles close to the torch axis, because a helical motion on the plasma jet by the vortex gas flow drags off the spray powder from the jet axis. Similarly, the stepped nozzle can reduce the gas swirl effect on the plasma jet. Because the stepped nozzle enhances turbulent effects behind the nozzle step, the helical motion of the plasma jet is disturbed. Therefore, homogenous treatment of particles in the plasma spraying can be achievable by using the stepped nozzle.

\section{CONCLUSION}

The geometrical effects of anode nozzle for the nontransferred torch on air entrainment are compared between the stepped and cylindrical nozzles by measurements of gas compositions using the quadruple mass spectrometry. As a result of the measurements of argon and air mole fractions in the plasma flame, the entrainment of ambient air appears to be reduced in the stepped nozzle case compared with the cylindrical one 
due to the relatively slower gradients of velocity around the peripheral region of plasma jet produced by the stepped nozzle.

The enthalpy probe method is used to find the effects of the entrainment of ambient air on the characteristics of plasma jets such as enthalpy, temperature, and velocity. Since the stepped nozzle yields a less air intrusion effect on the thermal plasma, it has produced the thermal plasma jets with higher enthalpy and temperature and more slowly varying axial velocity. The nozzle effects on air entrainment are found to be greatly different between the stepped and cylindrical nozzles mostly around the core region of plasma flames. Accordingly, the differences in enthalpy, temperature, and velocity between the stepped and cylindrical nozzle cases are observed mainly along the centerline of the thermal plasma jet.

From the results of this experimental work, a high quality of coating products is expected by using the stepped nozzle in plasma spraying, because the melting and accelerating processes of injected coating powders are strongly related to the enthalpy, temperature, velocity, and turbulence of the plasma flame. Suitable design requirements of the nozzle could be determined to produce the fast molten coating powders sprayed onto the substrate for an efficient process of plasma spraying.

\section{REFERENCES}

[1] J. R. Fincke, C. H. Chang, W. D. Swank, and D. C. Haggard, "Entrain and demixing in subsonic thermal plasma jets: comparison of measurements and predictions," Int. J. Heat Mass Transfer, vol. 37, pp. 1673-1682, 1994.

[2] A. Capetti and E. Pfender, "Probe measurements in argon plasma jets operated in ambient argon," Plasma Chem. Plasma Process., vol. 9, pp. 329-341, 1989.

[3] M. Jankovic and J. Mostaghimi, "A new nozzle design for dc plasma spray guns," Plasma Chem. Plasma Process., vol. 15, pp. 607-628, 1995.

[4] K. D. Kang and S. H. Hong, "Numerical analysis of shroud gas effects on air entrainment into thermal plasma jet in ambient atmosphere of normal pressure," J. Appl. Phys., vol. 85, pp. 6373-6380, 1999.

[5] E. Pfender, "Plasma jet behavior and modeling associated with the plasma spray process," Thin Solid Films, vol. 238, pp. 228-241, 1994.

[6] G. L. Brown and A. Roshko, "On density effects and large structure in turbulent mixing layers," J. Fluid Mech., vol. 64, pp. 775-816, 1974

[7] W. G. Hill Jr., R. C. Jenkins, and B. L. Gilbert, "Effects of the initial boundary-layer state on turbulent jet mixing," AIAA J., vol. 14, pp. 1513-1514, 1976

[8] W. D. Swank, J. R. Finke, and D. C. Haggard, "Modular enthalpy probe and gas analyzer for thermal plasma measurements," Rev. Sci. Instrum., vol. 64, pp. 56-62, 1993.

[9] M. Rahmane, G. Soucy, and M. I. Boulos, "Analysis of the enthalpy probe technique for thermal plasma diagnostics," Rev. Sci. Instrum., vol. 66, pp. 3424-3431, 1995.

[10] M. I. Boulos, P. Fauchais, and E. Pfender, Thermal Plasmas: Fundamentals and Applications. New York: Plenum, 1994, vol. 1, pp. 385-448.

[11] J. E. Broadwell and R. E. Breidenthal, "A simple model of mixing and chemical reaction in a turbulent shear layer," J. Fluid Mech., vol. 125, pp. 397-410, 1982

[12] R. Spores and E. Pfender, "Flow structure of a turbulent thermal plasma jet," Surface Coat. Technol., vol. 37, pp. 251-270, 1989.

[13] H. C. Chen, E. Pfender, and J. Heberlein, "Improvement of plasma spraying efficiency and coating quality," Plasma Chem. Plasma Process., vol. 17, pp. 93-105, 1997.

[14] J. R. Fincke, D. M. Crawford, S. C. Snyder, W. D. Swank, D. C. Haggard, and R. L. Williamson, "Entrainment in high-velocity, high-temperature plasma jets. Part 1: experimental results," Int. J. Heat Mass Transfer, vol. 46, pp. 4201-4213, 2003.

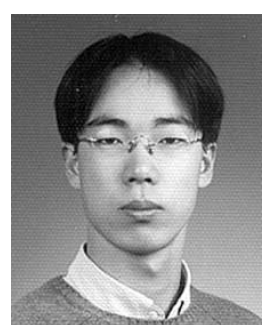

Sooseok Choi was born in Pohang, Korea, in 1979. $\mathrm{He}$ received the B.S. degree in nuclear engineering from Seoul National University, Seoul, Korea, in 2002.

In March 2002, he joined the Fusion and Plasma Application Laboratory, Department of Nuclear Engineering, Seoul National University. His research interests include diagnostics and applications of thermal plasmas.

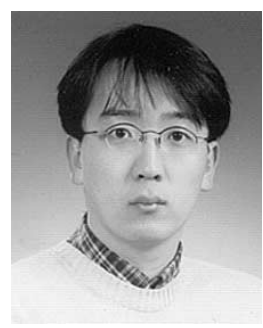

Tae Hyung Hwang received the B.S. and M.S. degrees in nuclear engineering from Seoul National University, Seoul, Korea, in 1997 and 1999, respectively.

His research interests include the diamond CVD by arc-jet thermal plasma, high-power plasma torch, and plasma diagnostics.

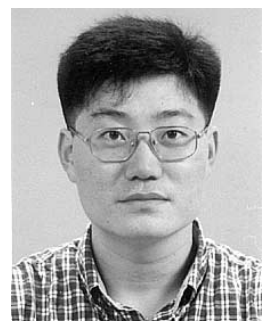

Jun Ho Seo received the B.S. and M.S. degrees in nuclear engineering from Seoul National University, Seoul, Korea, in 1994 and 1996, respectively. He is currently working toward the Ph.D. degree in the Department of Nuclear Engineering the same university.

His research interests include characterization of DC-RF hybrid plasma and plasma diagnostics using the enthalpy probe method.

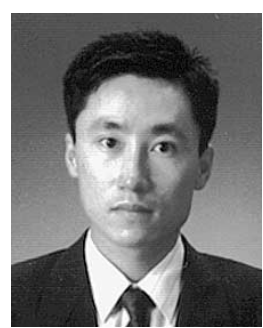

Dong Uk Kim received the B.S. and M.S. degrees in nuclear engineering from Seoul National University, Seoul, Korea, in 1991 and 1993, respectively.

His current research interests include materials processing using thermal plasmas and fabrication of solid oxide fuel cells.

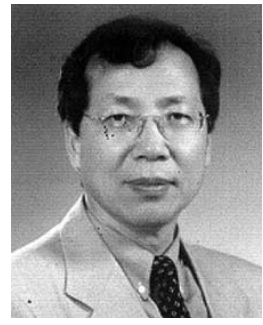

Sang Hee Hong ( ${ }^{\prime}$ '87) received the B.S. degree in applied physics from Seoul National University, Seoul, Korea in 1974. He received the M.S. and $\mathrm{Ph} . \mathrm{D}$. degrees in electrical engineering from Colorado State University, Fort Collins, in 1975 and 1978 , respectively.

In 1979, he joined the College of Engineering, Seoul National University, where he is currently a Professor of nuclear engineering. He spent a year at the University of Sydney, Sydney, Australia, in 1989 as a Visiting Professor. His research interests in the U.S. and Australia were stability analysis of MHD flows and theory of rotating plasma and plasma centrifuges. His research interests in Korea moved to numerical modeling of tokamak plasma and industrial processing plasmas. Recently, his efforts have been concentrated on tokamak plasma modeling and development of thermal plasma sources, such as dc and rf plasma torches and pulsed corona and dielectric barrier discharge reactors, as well as their materials and environmental processing. 